hallucinosis now ... her manner is much more normal, and her conversation is more coherent and reasonable. I think that she will soon be well enough to go on leave on trial."

\section{Summary and Conclusions}

A case is stated for the use of electrical convulsant therapy under out-patient conditions.

A portable apparatus suitable for such a purpose is described.

Certain modifications in technique and procedure associated with the use of this apparatus are set out, together with their advantages.

The clinical results so far obtained are regarded as encouraging, and will, it is hoped, be made the subject of a further communication.

\section{ERYTHROMELALGIA}

\author{
BY \\ E. D. TELFORD, B.Ch., F.R.C.S. \\ Emeritus Professor of Surgery, University of Manchester \\ AND

\section{H. T. SIMMONS, M.D., Ch.M., F.R.C.S.} \\ Research Fellow, Manchester Royal Infirmary \\ (From the Department for Clinical Research in Disorders \\ of the Autonomic Nervous System, Manchester \\ Royal Infirmary)
}

In 1878 Weir Mitchell described a condition of redness and burning pain of the extremities for which he coined the name "erythromelalgia." He considered the lesion to be a rare vasomotor neurosis of the extremities. The condition has since been described and discussed by a number of observers, and in reading these descriptions one cannot help being struck by the confusion which has arisena confusion so great that it has led some authors to doubt whether erythromelalgia is a definite disease. This confusion no doubt has its origin in the fact that a combination of redness and pain is a frequent concomitant of many different diseases of the peripheral circulation. We believe, however, from our experience that the lesion described by Weir Mitchell does exist as a separate entity -that is, it does occur in a pure form unaccompanied by any clinical signs of peripheral vascular disease. It is undoubtedly very rare, and in the course of nine years in a busy "neurovascular" clinic we have seen but four cases.

\section{Symptoms}

The patients are usually young adults, and the condition is probably more common in women. The attacks of redness and pain are brought on by warmth, by exercise, and by the dependent position of the limb. They may occur in the hands, but are more of ten seen in the feet. At first the trouble is most evident in the heat of summer, but as time goes on the attacks become more severe and last longer, occurring also in the winter. The pain is described as of a burning character, limited at first to the toes or to the heel, but later involving the whole foot. It is much aggravated by warmth, as of bedclothes, by the dependent position, and by walking. It tends to get worse, and may make the patient's life a well-nigh intolerable misery: one of our patients had spent six months in bed, unable to tolerate the lightest covering on the feet.

In an attack the feet are bright red and the skin may be extremely tender to the lightest touch ; the appearance of the foot is like the erythema of a burn of the first degree. We have not seen the throbbing arteries which Weir Mitchell, perhaps with a touch of drama, added to his list of physical signs; normal pulsation is, however, present throughout the foot. The temperature of the skin is raised during the attack. Elevation of the foot above the cardiac level lessens the symptoms and causes some diminution of the colour, but does not lead to any ischaemic pallor. Urticaria factitia has been described in some cases, but was not present in any of our four patients, nor did any of them show hyperidrosis.

\section{Pathology}

The cause of erythromelalgia is unknown. Weir Mitchell regarded it as a vasomotor neurosis. Buerger (1924) states that the sympathetic system is in all probability the seat of the disease, and this view receives some support from the immediate and complete cure which has in our hands followed lumbar cord ganglionectomy. It is also significant that in the recovery phase of a Raynaud attack, which is thought by most observers to be of sympathetic origin, the extremities involved are red and painful. Lewis (1933-4) regards the condition as a "susceptible state" in which the skin of certain patients reacts to a stimulus at a point well below the threshold of a normal individual. It is a common observation that some patients who experience trouble in the winter from erythrocyanosis or perniosis are apt to complain of sensitive burning feet in hot weather, but we have never seen this amount to anything more than a mild inconvenience. Collier (1898) thought that erythromelalgia was associated with nervous lesions, and considered that its presence might afford early indication of serious disease of the central nervous system. This view has, however, not been substantiated.

\section{Differential Diagnosis}

The condition which presents some superficial resemblance to erythromelalgia, and to which most of the confusion in the literature is due, is the painful rubor common to certain peripheral vascular diseases.

The Painful Rubor.-In cases of progressive obstruction of the peripheral arteries there comes a time when a reddish discoloration of the skin appears. This happens first in the digits and spreads later to the hand or foot. This rubor is of a dark brick-red colour, and is more evident in the dependent position. When the discoloured part is elevated above the level of the heart the rubor gives way to a cadaveric pallor, and the rapidity of the change in colour gives a direct measure of the degree of arterial obstruction. The earlier stages of rubor are not painful, but as the colour extends and deepens the pain begins. This pain, which is likely to become constant and severe, is a "rest pain," and is quite distinct from claudication, which is an "exertion pain." The rest pain is diminished by the dependent position, and these unfortunate patients try to gain sleep by hanging the limb over the side of the bed. Rest pain is no doubt due to tissue starvation, and it will disappear in dramatic fashion if a sympathectomy has been able to dilate a sufficient collateral supply. The condition of painful rubor is a grave one, and is to be regarded as a pre-gangrenous state.

There are many points of difference between painful rubor and the erythromelalgia of Weir Mitchell. Rubor is seen most commonly in young adult males (thromboangiitis obliterans) and in elderly men (arteriosclerosis). Pallor on elevation is not seen in erythromelalgia, and the pain in rubor is eased by the dependent position. Finally, the painful rubor is no more than a symptom of some grave underlying condition, which is always a threat to the limb and often to the life of the patient. 


\section{Treatment}

Treatment has hitherto been purely symptomatic and of little avail. In view of the probability that the disease is due to some disorder of the sympathetic nervous system, it would appear to be justifiable to treat the severe intractable cases by section of the sympathetic chain.

Since 1936 we have treated three cases by lumbar cord ganglionectomy; the fourth patient was not so treated because of a background of thyrotoxicosis. The results in the three operated patients have been excellent: in each case the symptoms have completely disappeared and the patient has remained well. Short notes of the cases submitted to operation are as follows:

Case 1.-A woman aged 24, a machinist, had had hot and burning feet when she was a girl at school. The trouble steadily got worse and the attacks began to come on in the winter. In these attacks, which lasted from three to four weeks and had intervals of only a few days between them, she says the pain was very severe and the feet "felt on fire." She could not walk, and had spent the previous six months lying in bed with the bare feet in the open. She was a thin pale woman, much worn by pain and loss of sleep. Both feet were bright red in colour-not sweating, very tender, with prominent veins; arterial pulsation was present but not exaggerated. A left-sided lumbar cord ganglionectomy was performed in July, 1936. The unilateral operation was done in order that the unoperated limb might serve as a control. The result in the left foot was entirely satisfactory. After watching the case for a year, the operation was done on the right side with equally good results. The patient has been seen since on many occasions, and remains entirely free from her former symptoms.

Case 2.-A man aged 55, a clerk, began to suffer five years ago from a hot patch on the right heel. This quickly spread to the whole foot, and shortly involved the left one. He then complained of burning pain, which varied little in summer or winter. He was quite incapacitated by the pain and tried to obtain sleep by bathing the feet in cold water two or three times during the night. There was no evidence of any disturbance in the blood supply to the feet; all pulses were present, but the skin was red, dry, intensely tender, and felt hot. A bilateral lumbar cord ganglionectomy was done in February, 1940, after which the symptoms at once disappeared; the feet returned to normal and he is perfectly well.

Case 3.-A woman aged 26, a supervisor, complained of burning pain in the feet, made much worse by warmth. The condition was typical of erythromelalgia. She was kept under observation for twelve months, during which time she became worse. A bilateral cord ganglionectomy was done in July, 1940. The pain was at once abolished and the feet became normal. She was seen a few days ago, and remains quite free from her trouble.

\section{Summary}

An account of erythromelalgia is given and its diagnosis from painful rubor is discussed.

Treatment by sympathectomy is advised and records of three successful cases are given.

\section{REFERENCES}

Buerger, L. (1924). The Circulatory Disturbances of the Extremities, Saunders, Philadelphia.

Collier, Lancet, 2, 401.

Lewis, Sir T. (1933-4). Clin, Sci., 1, 175.

Mitchell, S. Weir (1878). Amer. J. med. Sci., 76. 17.

J. G. Rushton, R. W. Cragg, and L. K. Stalker (Arch. intern. Med., 1940, 66, 531) give an account of a young woman who suffered from spontaneous attacks of hypoglvcaemia. Operation failed to reveal the pancreatic tumour which was expected. The patient died after the operation, and necropsy showed extreme atrophy of the adrenal glands and "extensive inflammatory destruction of the thyroid of the type described by Hashimoto." No tumour of the pancreas was found, and the authors consider the hypoglycaemia to have been due to the adrenal changes.

\section{REVERSED ROTATION OF THE INTESTINE ACCOMPANIED BY ENCAPSULATING PERITONITIS * REPORT OF A CASE}

\author{
BY
}

\author{
J. W. GEARY GRANT, F.R.C.S. \\ Coisulting Surgeon, Royal Infirmary, Cardiff
}

The occurrence in the same patient of the above two conditions, which are decidedly rare and are liable to cause some embarrassment to the operator, appears to be worth recording.

\section{Case Report}

On May 5, 1939. assisted by Dr. R. Nelson of Treherbert, 1 operated on a patient of his, a miner aged 62 . The history given was that two days previously he was seized with sudden abdominal pain followed by complete constipation. The abdomen was moderately distended. Nothing was felt per rectum, and the history was negative. Under general anaesthesia the abdomen was opened by a right paramedian incision. All that was at first seen was a large cyst with smooth shining white walls; it resembled an ovarian cyst, and occupied the greater part of the left hypochondrium, epigastrium, and left lumbar region (Fig. 1). The only portion

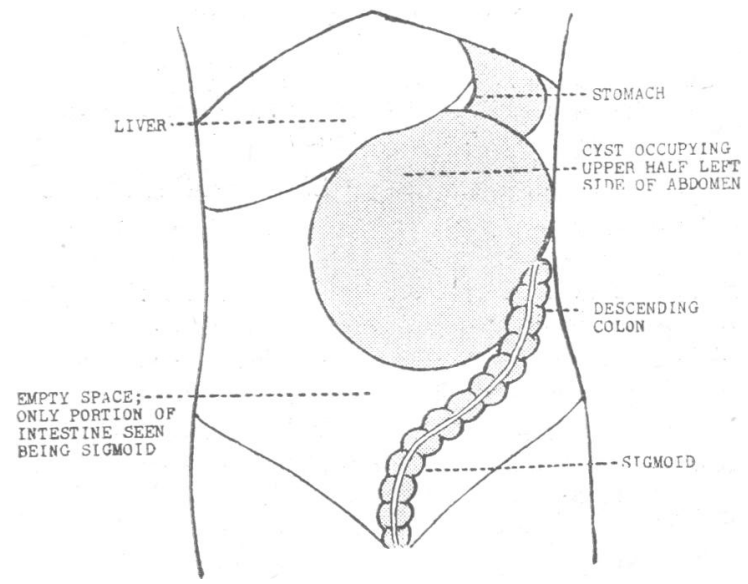

FIG. 1.-Diagram of condition found at first operation for acute intestinal obstruction.

of the intestines visible was the descending colon, which appeared to come out from under the cyst in the left hypochondrium. The rest of the abdomen being carefully walled off, a small incision was made in the cyst, and out of this a quantity of foul-smelling blood-stained fluid escaped. On enlarging the opening it was found to be occupied by the whole of the intestine from the duodenum to the left colon, which emerged in the left hypochondrium from the sac. The gut was entirely free from the inner wall of the sac, and no difficulty was experienced in cutting away the sac from its attachment to the posterior abdominal wall. There was then found to be a volvulus of the mid-gut loop, which had undergone rotation of about 270 degrees in a clockwise direction. This was undone. The distended caecum showed a gangrenous patch the size of a shilling a Paul's tube was fixed in this opening and the caecum was fixed in the abdominal wound, which was closed.

The abdominal wound healed after some suppuration, but a large opening in the caecum remained from which all the intestinal contents escaped. The man's condition having greatly improved, an attempt was made to close the caecostomy; this was immediately followed by symptoms of obstruction. It was then decided to reopen the abdomen. On Aurgust 2, the opening in the caecum being temporarily closed, the abdomen was opened under general anaesthesia. It was found that the caecum and ascending colon came out

* Abstract of a lecture given in the Welsh National School of Medicine. 